\title{
Analysis of transverse beam stabilization with radio frequency quadrupoles
}

\author{
M. Schenk, ${ }^{1,2, *}$ A. Grudiev, ${ }^{1}$ K. Li, ${ }^{1}$ and K. Papke ${ }^{1}$ \\ ${ }^{1}$ CERN, CH-1211 Geneva, Switzerland \\ ${ }^{2}$ EPFL, CH-1015 Lausanne, Switzerland \\ (Received 7 June 2017; published 13 October 2017)
}

\begin{abstract}
A radio frequency ( $\mathrm{rf}$ ) quadrupole has been considered as a potential alternative device for Landau damping in circular hadron colliders. The objective of this study is to benchmark and confirm its stabilizing effect predicted by stability diagram theory by means of numerical tracking simulations. To that end, two complementary models of the device are implemented in PyHEADTAIL, a 6D macroparticle tracking code designed to study the formation and mitigation of collective instabilities. The rf quadrupole model is applied to a slow head-tail instability observed experimentally in the Large Hadron Collider to show that such a device can in principle provide beam stability similarly to magnetic octupoles. Thereafter, alternative usage schemes of rf quadrupoles also in combination with magnetic octupoles are proposed, discussed, and benchmarked with simulations.
\end{abstract}

DOI: 10.1103/PhysRevAccelBeams.20.104402

\section{INTRODUCTION}

To improve the luminosity of future machines like the High Luminosity Large Hadron Collider (HL-LHC) or the Future Circular Collider (FCC), the brightness of the particle beams will be significantly increased [1,2]. This is realised by operating the machine with a higher bunch intensity and lower transverse emittances. These parameters and therefore the maximum achievable luminosity will be strongly limited by the presence of transverse collective beam instabilities induced by the transverse beam coupling impedance of the accelerator ring.

A powerful stabilizing mechanism against this type of instabilities is the effect of Landau damping which is present when there is an incoherent spread in the betatron frequencies, or tunes, of the particles in the beam $[3,4]$. The tune spread is a result of nonlinearities in the machine. Partially, they are of parasitical nature, e.g., originating from non-linear space-charge forces, nonlinearities in the magnetic focusing systems, beam-beam interactions at collision, etc. In addition, however, they are often introduced by design through dedicated nonlinear elements for better control and efficiency. Magnetic octupoles are commonly used for the latter purpose. In the Large Hadron Collider (LHC), families of 84 focusing and 84 defocusing, $0.32 \mathrm{~m}$ long superconducting magnetic octupoles are installed to produce an incoherent betatron tune spread that depends on the transverse amplitudes, or actions, $\left(J_{x}, J_{y}\right)$ of the particles in the beam [5]. These

*michael.schenk@cern.ch

Published by the American Physical Society under the terms of the Creative Commons Attribution 4.0 International license. Further distribution of this work must maintain attribution to the author(s) and the published article's title, journal citation, and DOI. so-called Landau octupoles are an integral part of the LHC instability mitigation toolset and are extensively used for beam stabilization during operation [6,7].

Future hadron colliders will operate with beams of smaller transverse emittances making the Landau octupoles significantly less effective due to the reduced spread in $\left(J_{x}, J_{y}\right)$. This effect is even more pronounced at higher beam energies as a result of adiabatic damping and may eventually lead to a loss of Landau damping of potentially performance-limiting collective instabilities. Alternative approaches, such as betatron detuning with longitudinal action $J_{z}$ are currently under study. Longitudinal action provides a much larger handle for introducing a betatron tune spread due to the orders of magnitude larger spread in $J_{z}$ compared to $\left(J_{x}, J_{y}\right)$ of the beams of high energy hadron colliders [8]. The basic formalism for this particular stabilizing mechanism has been developed by J. Scott Berg and F. Ruggiero [9]. With their work they demonstrate that the tune spread introduced as a function of $J_{z}$ improves the stability of particle beams. The underlying mechanism is similar to the Landau damping effect from magnetic octupoles.

A radio frequency (rf) quadrupole has been considered as a potential device to realise betatron detuning with longitudinal amplitude [8]. Analytical calculations predict that the maximum RMS betatron tune spread generated by the LHC Landau octupoles can theoretically be achieved with a few metres long superconducting $\mathrm{rf}$ device operating in a transverse magnetic quadrupolar mode. This is for LHC nominal beam and machine parameters at top energy of $7 \mathrm{TeV}$. For comparison, the total active length of the LHC Landau octupoles is about $56 \mathrm{~m}$.

The main purpose of this study is to confirm the stabilizing effect of an rf quadrupole predicted by stability diagram theory with numerical simulations using the 
PyHEADTAIL 6D macroparticle tracking code [10]. Section II summarizes the working principle of an $\mathrm{rf}$ quadrupole, describes possible designs and the main characteristics of the device, potential benefits over conventional stabilizing methods, as well as the existing theory of the stabilizing mechanism. Section III briefly introduces the basic principles of the PyHEADTAIL code and details on the implemented rf quadrupole models. Section IV contains the numerical proof for beam stabilization with an $\mathrm{rf}$ quadrupole. The study is based on a slow head-tail instability that is observed in the LHC and proven to be Landau damped by means of the magnetic octupoles both experimentally and in simulations [11]. Here, tracking studies show that an rf quadrupole manages to stabilize the beam in a similar manner. Section $V$ discusses the beam dynamics in presence of an rf quadrupole in more detail. Furthermore, it introduces various schemes for the use of $\mathrm{rf}$ quadrupoles, among them a two-family configuration as well as the combination of an rf quadrupole with magnetic octupoles. The schemes are partially motivated by stability diagram theory and are benchmarked with tracking simulations.

\section{RADIO FREQUENCY QUADRUPOLE}

This section gives a brief overview on the main aspects of an rf quadrupole device. It discusses first two cavity designs explaining the main differences between the two geometries as well as the relevant parameters that have been optimized $[8,12]$. The basic working principle of the device is then explained by deriving an analytical formula for the incoherent betatron detuning that it generates. Subsequently, the features and potential advantages over conventional stabilizing methods are discussed in more detail in terms of the mitigation of collective instabilities. Finally, an existing, approximate, stability diagram theory is applied for a qualitative characterization of the Landau damping [9].

\section{A. Device description}

The purpose of an rf quadrupole is to produce transverse quadrupolar kicks on the particles in the bunch with a strength that depends on the longitudinal coordinate. Every particle feels a different focusing (defocusing) force as it passes through the device, and hence experiences a change in the betatron tunes depending on its longitudinal position in the bunch. This results in an incoherent betatron tune spread which can produce Landau damping. The frequency of the rf quadrupole described in this study is chosen to be $800 \mathrm{MHz}$ for two reasons. First, the bunches of the HL-LHC and FCC-hh (hadron-hadron) are foreseen to have a length of the order of $\sigma_{z} \approx 0.1 \mathrm{~m}[1,2]$. An $\mathrm{rf}$ quadrupole operating at $800 \mathrm{MHz}$ has an rf wave length that matches the bunch length and provides the best beam stabilizing efficiency according to numerical simulations [12]. Second, for practical reasons, the rf quadrupole
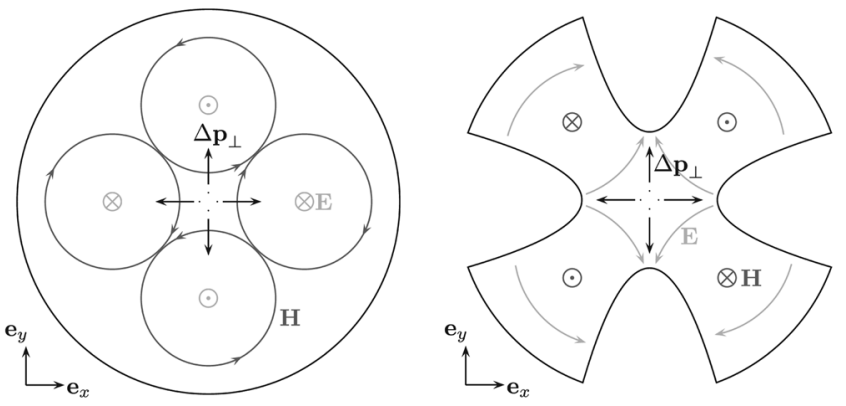

FIG. 1. Qualitative description of the quadrupolar fields leading to a transverse kick on a charged particle (cross-section). The direction of the momentum change $\Delta \mathbf{p}_{\perp}$ is indicated for a positively charged particle. Left: Elliptical cavity type operating in a TM mode. Right: Four-vane cavity operating in a TE mode.

frequency is limited to harmonics of the main rf system (400 MHz) of the aforementioned machines.

In [12], two different cavity designs for a superconducting rf quadrupole have been proposed and thoroughly optimized primarily for quadrupolar field strength, but also in terms of transverse and longitudinal beam coupling impedance, and peak electric $E_{p k}$ and magnetic $B_{p k}$ surface fields. While the first type is an elliptical cavity operating in a transverse magnetic (TM) quadrupolar mode, the second one is a four-vane cavity operating in a transverse electric (TE) quadrupolar mode. The geometry of the latter was motivated by the rf quadrupole (RFQ) linac [13]. Its design was chosen to reach higher quadrupolar field strengths at smaller cavity size. A cross section of the quadrupolar fields for the two cavity types is given in Fig. 1. The transverse kicks $\Delta \mathbf{p}_{\perp}$ on a positively charged particle passing the device off-axis at a specific moment in time are indicated by the black arrows.

The goal of the cavity optimization procedure is mainly to find a geometry that provides the largest quadrupolar field strength at the lowest surface fields and impedance. For the elliptical cavity, the $\mathrm{TM}_{210}$ mode is the best for that purpose. An illustration of the normalized magnetic (left) and electric (right) field distributions for this particular mode is given in the upper part of Fig. 2 showing one octant of the elliptical cavity. The geometry and field distributions of the four-vane cavity are depicted in the lower part of Fig. 2. The main advantages of the four-vane cavity compared to an elliptical one are [12]: (i) the quadrupolar field strength per cavity is up to two to five times larger than that of the elliptical one, given that the aperture has a radius $<50 \mathrm{~mm}$, and (ii) the four-vane cavity is more compact and requires hence smaller cryomodules and less cooling power. As a result, the number of cavities can be reduced and therewith the overall impedance and cost of the system.

\section{B. Working principle}

In the thin-lens approximation, an ultrarelativistic particle of index $i$, electric charge $q$, and momentum $p$ 

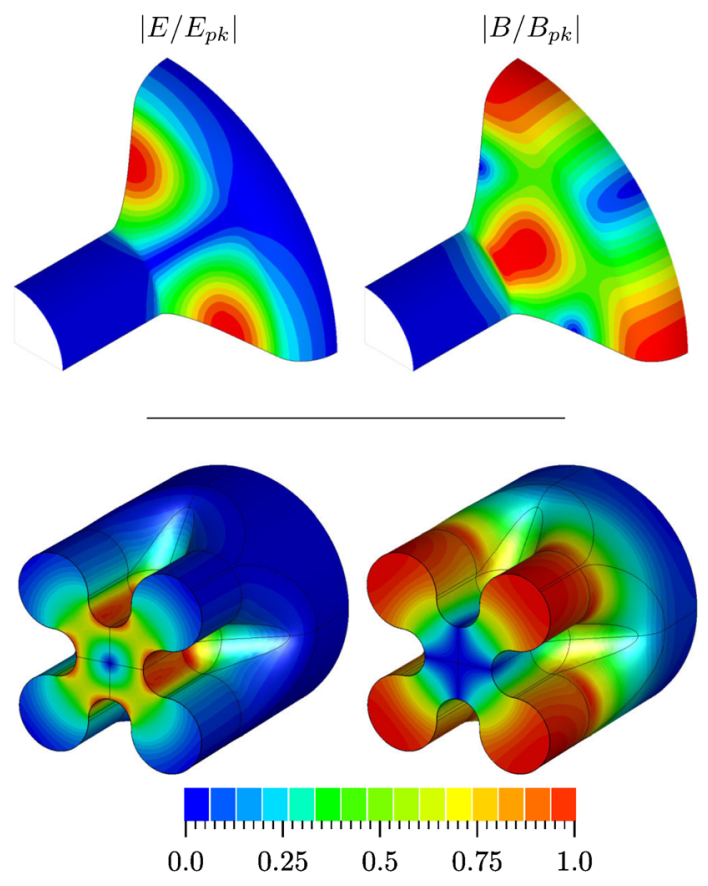

FIG. 2. Normalized electric (left) and magnetic (right) fields for an octant of the elliptical (top) and for half of the four-vane (bottom) cavity respectively.

traversing an rf quadrupole along the $z$-axis experiences both transverse and longitudinal kicks

$$
\begin{aligned}
\Delta \mathbf{p}_{i}^{\perp} & =p k_{2}\left(y_{i} \mathbf{e}_{y}-x_{i} \mathbf{e}_{x}\right) \cdot \cos \left(\omega t_{i}+\varphi_{0}\right), \\
\Delta p_{i}^{\|} & =-\frac{\omega}{2 \beta c} p k_{2}\left(x_{i}^{2}-y_{i}^{2}\right) \cdot \sin \left(\omega t_{i}+\varphi_{0}\right),
\end{aligned}
$$

where $\omega$ denotes the rf quadrupole angular frequency, $\mathbf{e}_{x}$ and $\mathbf{e}_{y}$ are the unit vectors along the $x$ and $y$ coordinate respectively, $\varphi_{0}$ is a constant phase offset, and $t_{i}$ is the time when the particle traverses the rf quadrupole. The latter is defined with respect to the particle which is at the zerocrossing of the rf quadrupole voltage, and $t_{i}=0$ coincides with the bunch center. This assumption is made throughout. The substitution $t_{i}=z_{i} / \beta c$ gives the longitudinal dependence of the quadrupolar kick strength along the particle bunch, where $\beta$ is the relativistic beta and $c$ the speed of light. The parameter $k_{2}$ refers to the amplitude of the normalized integrated quadrupolar gradient, i.e., it accounts for the transit time factor,

$k_{2}=\frac{q}{\pi r p c} \int_{0}^{2 \pi}\left\|\int_{0}^{L}\left(E_{x}-c B_{y}\right) e^{i \omega z / c} d z\right\| \cos \varphi d \varphi$,

where $L$ is the length of the rf quadrupole cavity, and $[r, \varphi, z]$ are the cylindrical coordinates. Throughout this article $k_{2}$ is expressed in magnetic units $[\mathrm{Tm} / \mathrm{m}]$ using the conversion $b^{(2)}=B_{0} \rho k_{2}$, where $B_{0} \rho$ is the magnetic rigidity of the beam.
Depending on their longitudinal position, particles traversing the rf quadrupole experience a different quadrupolar (de-)focusing force and hence a change in their betatron tunes

$$
\Delta Q_{x, y}^{i}= \pm \beta_{x, y} \frac{b^{(2)}}{4 \pi B_{0} \rho} \cos \left[\frac{\omega z_{i}}{\beta c}+\varphi_{0}\right],
$$

where $\beta_{x, y}$ are the transverse beta functions of the machine lattice at the location of the quadrupole kicks in Eq. (1). Equation (4) describes the detuning that a particle $i$ experiences from a single passage through the rf quadrupole according to its current longitudinal position $z_{i}$ in the bunch. As the particle undergoes synchrotron motion, its longitudinal position changes turn after turn. Hence, each time it passes through the device, it experiences a different kick and hence a different detuning. Given enough time, the longitudinal turn-by-turn position of the particle will be evenly distributed over the interval $\left[-\hat{z}_{i}, \hat{z}_{i}\right]$, where $\hat{z}_{i}$ is its maximum synchrotron oscillation amplitude. This is true as long as the synchrotron tune is not a rational number. Figure 3 illustrates the situation for two different rf
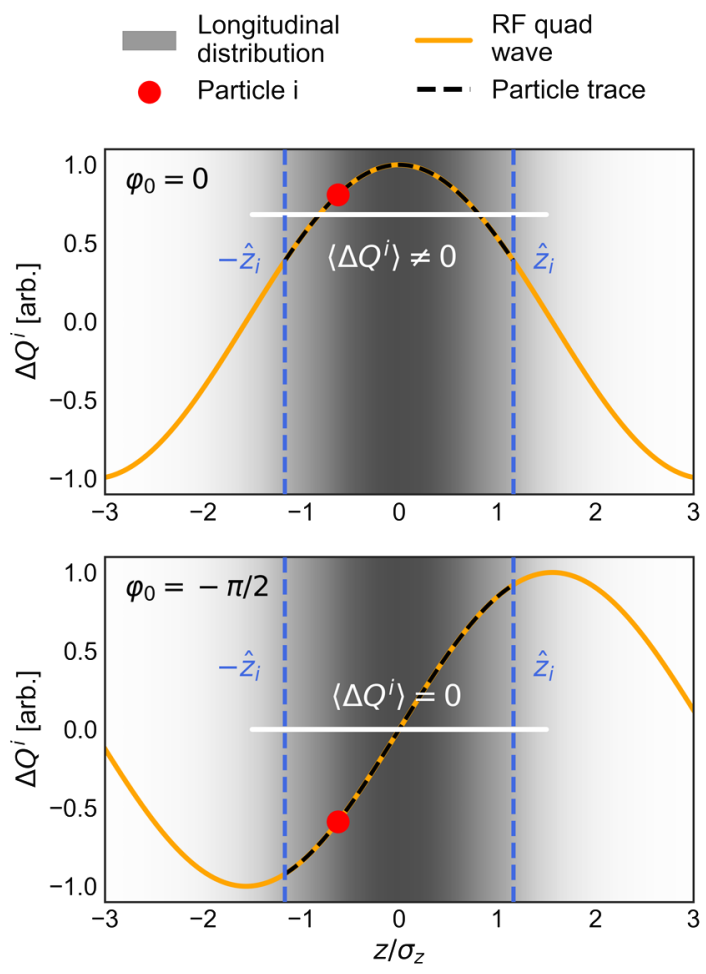

FIG. 3. Illustration of the detuning that a particle (red) experiences as it passes through an rf quadrupole (orange) turn after turn. Given enough time, the longitudinal position of the particle will be evenly distributed over the interval $\left[-\hat{z}_{i}, \hat{z}_{i}\right]$ due to the synchrotron motion. It will hence experience a changing detuning every time it passes through (black dashed curve). If the rf quadrupole phase is $\varphi_{0}=0$ (top), the average detuning will not vanish and there is a net incoherent tune spread. If $\varphi_{0}=-\pi / 2$ there will be no effective tune spread. 
quadrupole phase offsets $\varphi_{0}=0$ (top) and $\varphi_{0}=-\pi / 2$ (bottom). The grey background represents the particle distribution (Gaussian) in the longitudinal plane. One particle (red) is observed over time as it passes through the rf quadrupole (orange) turn after turn. At every passage it experiences a detuning according to its current longitudinal position. The black dashed line corresponds to the interval $\left[-\hat{z}_{i}, \hat{z}_{i}\right]$ that the particle eventually traces out as it passes through the rf quadrupole for many turns, i.e., considering a long enough time (several synchrotron periods). It is worth noting that the situation is analogous for detuning with transverse action, such as from magnetic octupoles [14]. The only difference is that the betatron rather than the synchrotron motion is relevant. It is clear from Fig. 3 that for $\varphi_{0}=0$, the average detuning of the particles is nonzero which corresponds to a nonvanishing incoherent tune spread. For $\varphi_{0}=-\pi / 2$, however, the average detuning vanishes for all the particles and there is no net tune spread. To maximize hence the achievable incoherent betatron tune spread and to prevent it from averaging out over time, the rf quadrupole is operated with a phase of $\varphi_{0}=0$ or $\varphi_{0}=\pi$. This is an essential requirement for the Landau damping mechanism to work against the slow head-tail instabilities which develop over time scales of many synchrotron periods [4]. Selecting $\varphi_{0}=0$ means that the device is focusing (defocusing) in the horizontal (vertical) plane for particles in the bunch center $z_{i}=0$. The situation is inverse for $\varphi_{0}=\pi$. Equivalently, operating the cavity in one of these two modes means that particles located in the bunch center enter the device (anti-) on-crest of the rf wave. This is different from earlier studies $[15,16]$ where an $\mathrm{rf}$ quadrupole was proposed to increase the threshold of the transverse mode coupling instability (TMCI) [4]. In that case, the cavity would have to be operated at the zero-crossing of the rf voltage, i.e. with $\varphi_{0}= \pm \pi / 2$. This has an effect similar to the odd orders of chromaticity.

In the following we set $\varphi_{0}=0$. Equation (4) can be expanded into a Taylor series in $z_{i}$

$$
\Delta Q_{x, y}^{i}= \pm \beta_{x, y} \frac{b^{(2)}}{4 \pi B_{0} \rho}\left[1-\frac{1}{2}\left(\frac{\omega z_{i}}{\beta c}\right)^{2}+\mathcal{O}\left(z_{i}^{4}\right)\right] .
$$

Given that the wavelength of the rf wave is much larger than the bunch length $\sigma_{z}$, i.e. $\omega \sigma_{z} / \beta c \ll 1$, the higher order terms $\mathcal{O}\left(z_{i}^{4}\right)$ can be neglected. Assuming linear synchrotron motion and taking the average detuning over a long period of time (several synchrotron periods), again in analogy to the derivation of detuning with transverse action from magnetic octupoles [14], it is easy to show that the rf quadrupole indeed changes the betatron tunes as a function of the longitudinal action $J_{z}^{i}$ of a particle

$$
\left\langle\Delta Q_{x, y}^{i}\right\rangle \approx \pm \beta_{x, y} \frac{b^{(2)}}{4 \pi B_{0} \rho}\left[1-\frac{1}{2}\left(\frac{\omega}{\beta c}\right)^{2} \beta_{z} J_{z}^{i}\right] .
$$

$\beta_{z}$ has been defined as $\beta_{z} \doteq \eta R / Q_{s}$, where $\eta$ denotes the slip factor, $R$ is the accelerator ring radius, and $Q_{s}$ is the synchrotron tune. The longitudinal action is defined as $J_{z}^{i}=\left(z_{i}^{2}+\beta_{z}^{2} \delta_{i}^{2}\right) / 2 \beta_{z}$, where $\delta_{i}$ is the relative momentum deviation. The constant quadrupolar detuning term in Eq. (6) does not contribute to the effective tune spread as it affects all the particles in the same manner. The pure incoherent detuning of the particles with respect to each other is hence approximatively given by

$$
\begin{aligned}
\Delta Q_{x, y}\left(J_{z}\right) & \approx \mp \beta_{x, y} \frac{b^{(2)}}{8 \pi B_{0} \rho} \frac{\omega^{2}}{\beta^{2} c^{2}} \beta_{z} J_{z} \\
& =\mp a_{z}^{x, y} J_{z},
\end{aligned}
$$

where all the constants are combined in the parameter $a_{z}^{x, y}$ called detuning coefficient. The approximation is valid as long as $\omega \sigma_{z} / \beta c \ll 1$. Furthermore, it assumes that the rf quadrupole is operating with a phase $\varphi_{0}=0$. By changing the phase to $\varphi=\pi$, the signs flip, meaning that the tune spreads between the two transverse planes are swapped.

The longitudinal kick in Eq. (2), in a similar manner leads to an incoherent synchrotron detuning. This effect, however, can be neglected given the parameters of the particle colliders under consideration [8].

For the following discussion and in comparison to Eq. (7) it is useful to recall the transverse detuning with amplitude introduced by magnetic octupoles

$$
\begin{aligned}
& \Delta Q_{x}\left(J_{x}, J_{y}\right)=a_{x x} J_{x}+a_{x y} J_{y} \\
& \Delta Q_{y}\left(J_{x}, J_{y}\right)=a_{y y} J_{y}+a_{y x} J_{x},
\end{aligned}
$$

where $a_{i j}, i, j \in\{x, y\}$, are the detuning coefficients. They depend on the integrated octupolar field strength weighted with the beta functions at the location of the magnets around the accelerator ring and are inversely proportional to the magnetic rigidity of the beam [17]. Note that the two cross-detuning coefficients are identical, i.e. $a_{x y}=a_{y x}$.

\section{Features, potential advantages, and applications}

Figure 4 compares two different incoherent betatron tune distributions in the transverse tune space $Q_{x}$ vs. $Q_{y}$, averaged over several synchrotron periods. First, from magnetic octupoles which generate detuning with transverse action (blue) as in Eq. (8), and second, from a single rf quadrupole with $\varphi_{0}=0$ which generates detuning with longitudinal action (red) given by Eq. (7). Here, the magnetic octupoles are powered in a two-family scheme with focusing and defocusing elements. This scheme is applied for example in the LHC to optimize the beam stabilizing efficiency in both transverse planes [17]. When powered in such a way, magnetic octupoles produce a twodimensional tune footprint in $\left(Q_{x}, Q_{y}\right)$-space as shown in Fig. 4. The distribution is obtained assuming negative (positive) polarity of the focusing (defocusing) family. 


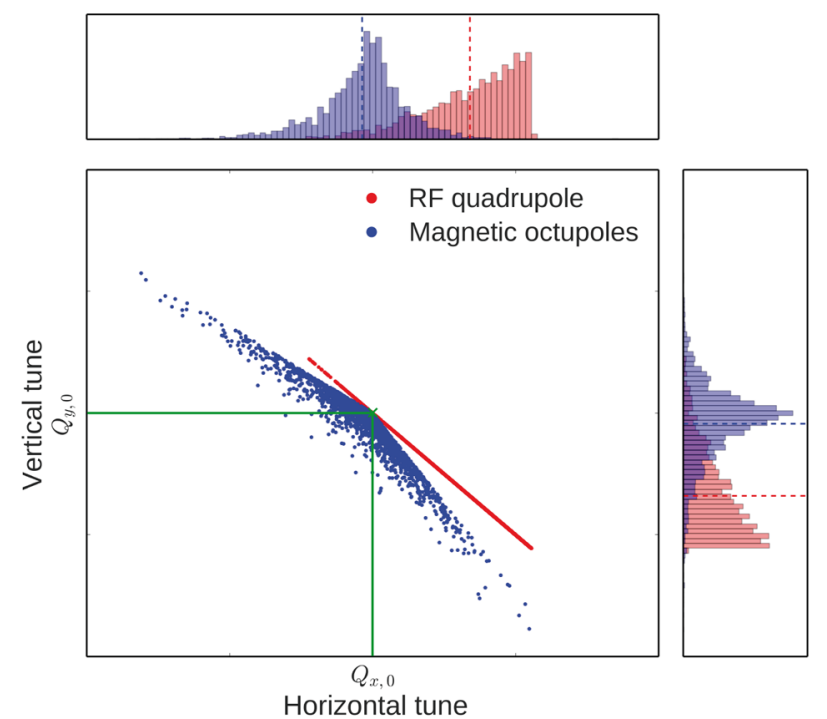

FIG. 4. Comparison of incoherent betatron tune distributions in the $\left(Q_{x}, Q_{y}\right)$-space introduced by magnetic octupoles (blue) powered in a two-family scheme with negative (positive) polarity in the focusing (defocusing) family, and a single rf quadrupole (red). The lines and the cross in green mark the bare machine tunes $\left(Q_{x, 0}, Q_{y, 0}\right)$. Histograms on the top and on the side show the projections of the tune distributions onto the $Q_{x}$ - and the $Q_{y}$-axis respectively.

The projection of the tune distribution onto the $Q_{x^{-}}\left(Q_{y^{-}}\right)$ axis is nearly symmetric with respect to the bare machine tune $Q_{x, 0}\left(Q_{y, 0}\right)$ identified with the green lines.

For the rf quadrupole the tune changes in $Q_{x}$ and $Q_{y}$ are fully correlated. This is explained by the fact that the detuning is dependent on only one variable $J_{z}$. The tilt of the distribution from the rf quadrupole is determined by the ratio of $\beta_{x}$ vs. $\beta_{y}$ at the location of the device [see Eq. (7)]. Here, the transverse beta functions are assumed to be identical. The tune projections for a single rf quadrupole are strongly asymmetric. This has important consequences for the stabilizing mechanism in the two transverse planes as discussed in more detail in Sec. II D. In addition to the incoherent tune spread, the rf quadrupole also introduces a constant, quadrupolar tune shift which is the same for all the particles. This is understood formally from the presence of the zeroth-order term in Eq. (5). Such an effect can be compensated with a dedicated quadrupole magnet if needed.

Figure 5 compares the transverse RMS tune spreads produced by an rf quadrupole (red) and the LHC Landau octupoles (blue) as a function of the beam energy for LHC design beam parameters $[5,17]$. The comparison is made using an $800 \mathrm{MHz}$ rf quadrupole installed at $\beta_{x, y}=200 \mathrm{~m}$. The strength is fixed at $b^{(2)}=0.22 \mathrm{Tm} / \mathrm{m}$ and is chosen in such a way as to provide the same RMS tune spread as the LHC Landau octupoles powered at their maximum current of 550A at top beam energy of $7 \mathrm{TeV}$ (green dashed line).
The strengths of both the rf quadrupole and the magnetic octupoles are held constant as a function of the beam energy. While the LHC Landau octupoles have a total active length of $56 \mathrm{~m}$, the equivalent rf quadrupole strength can theoretically be achieved with just one four-vane cavity with a total active length of $0.2 \mathrm{~m}$ to $0.3 \mathrm{~m}$ [12]. For this particular case, the active length of the rf quadrupole compared to magnetic octupoles is hence shorter by a factor 180 to 280 . The reason behind the much larger efficiency of an $\mathrm{rf}$ quadrupole in terms of detuning is the fact that the spread in $J_{z}$ is several orders of magnitude larger than that in $\left(J_{x}, J_{y}\right)$. This is generally true for the beams of high energy hadron colliders like LHC, HL-LHC, or FCC-hh. Specifically, for LHC design beam parameters at an energy of $7 \mathrm{TeV}$ there is roughly a factor $10^{4}$ difference in the transverse and the longitudinal action spreads. The main reason why the difference in active lengths between the rf quadrupole and magnetic octupoles does not reach a factor $10^{4}$, but rather is limited to 180 to 280 at the beam energy of $7 \mathrm{TeV}$, is that the transverse deflecting kicks in an rf cavity are much smaller than those in a superconducting magnetic octupole. This becomes evident when comparing the detuning coefficients of an $\mathrm{rf}$ quadrupole with those of magnetic octupoles. In this particular case and for the given beam energy, the former is $a_{z}^{x, y} \approx 16$, while the latter are of the order of $10^{5}$ for the LHC Landau octupoles at maximum strength [18]. The ratio between the two detuning coefficients amounts to a factor $\approx 10^{4}$ which, as expected, corresponds to the difference in the action spreads between the longitudinal and the transverse planes.

The advantages of producing a tune spread with an rf quadrupole rather than with magnetic octupoles become more pronounced when the beam energy increases as demonstrated by Fig. 5. Two effects play an important role here. First, due to the increased beam rigidity accounted for by the detuning coefficients, the effect of

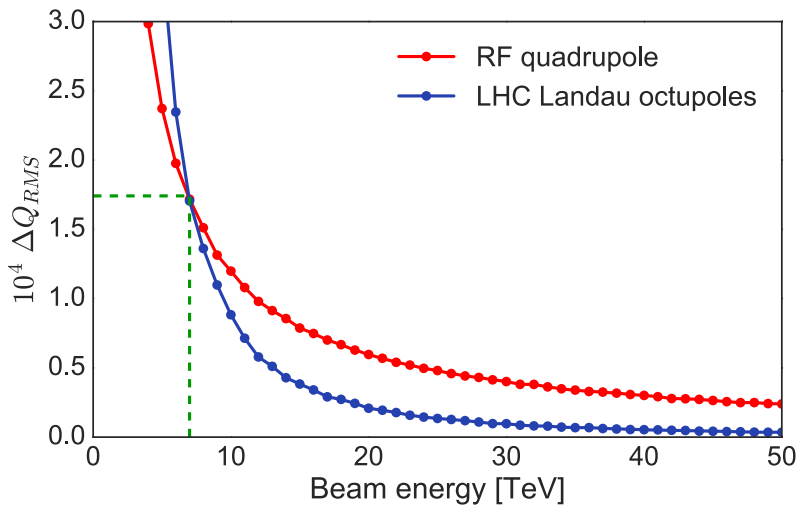

FIG. 5. Dependence of the RMS tune spread on the beam energy for both LHC magnetic octupoles at maximum strength (blue) and for an rf quadrupole (red), designed in such a way as to produce the same RMS tune spread at $7 \mathrm{TeV}$ (green dashed line). 
the kicks from both magnetic octupoles and rf quadrupole is reduced by $1 / \gamma$ (Lorentz factor). This directly translates into a reduction of the RMS tune spread by the same factor. Second, the spreads in respectively the transverse and the longitudinal actions exhibit a different dependence on the beam energy. The longitudinal beam stability in a machine like the LHC strongly relies on Landau damping in the longitudinal plane [19]. To keep enough stability margin during the energy ramp, the longitudinal emittance needs to be blown up. As a result, the longitudinal action spread is roughly held constant as the beam energy increases. On the other hand, the transverse action spreads suffer from adiabatic damping and decrease as $1 / \gamma$. To summarize, the RMS tune spread produced by the rf quadrupole is only reduced as $1 / \gamma$ (beam rigidity) while the one introduced by magnetic octupoles decreases as $1 / \gamma^{2}$ (beam rigidity and adiabatic damping). These considerations are based on the assumption that the machine optics remain unchanged during the energy ramp.

In addition to the improved detuning efficiency compared to magnetic octupoles, the performance of the $\mathrm{rf}$ quadrupole also remains unaffected by beam manipulations in the transverse plane, such as beam halo cleaning through collimation. During this process, the large transverse amplitude particles are removed from the beam in a controlled manner for reasons of machine protection $[1,5]$.

The rf quadrupole finds a potential application predominantly in future high energy hadron colliders, such as HL-LHC or FCC-hh, where the differences between the transverse and longitudinal emittances are most pronounced. Due to the small transverse emittance of the beams expected in these machines, the number of magnetic octupoles required to provide the tune spread for sufficient Landau damping is large, and a solution relying only on magnetic octupoles may be less cost-effective than an rf quadrupole, or a combination of the two elements. However, it is worth pointing out that the size of the incoherent betatron tune spread alone does not make a complete statement about the effectiveness of the Landau damping itself. The stabilizing mechanisms from detuning with transverse and longitudinal action respectively are different as seen from stability diagram theory explained in the following. Generally speaking, tracking simulations are the most accurate way at present to study the effectiveness of beam stabilization with an $\mathrm{rf}$ quadrupole.

\section{Stability diagram theory}

A practical approach to quantify the Landau damping generated by an incoherent tune spread is to use stability diagram theory. A stability diagram marks the area in the complex coherent tune shift plane $\operatorname{Re}\left(\Delta Q_{\text {coh }}\right)$ vs. $-\operatorname{Im}\left(\Delta Q_{\text {coh }}\right)$ for which Landau damping is effective. The real part of the complex coherent tune shift is obtained from a frequency analysis of the bunch centroid motion. The imaginary part corresponds to the growth rate of the instability at hand. For magnetic octupoles, the stability diagram theory is well established (see, e.g., [9]). The goal of this section is to summarize the stability diagram theory that exists for detuning with longitudinal amplitude, as introduced for instance by an rf quadrupole.

The top plot in Fig. 6 shows the incoherent tune distributions in the two transverse planes obtained analytically for a Gaussian beam for a single rf quadrupole with $\varphi_{0}=0$ assuming identical beta functions. In accordance with Eq. (7), the particles experience negative (positive) detuning in the horizontal (vertical) plane. The distributions in the two planes are strictly asymmetric and are mirrored with respect to each other. This is a direct consequence of the quadrupolar nature of the kicks introduced by the device. To obtain the distributions for $\varphi_{0}=\pi$, the labels of the two curves can simply be exchanged.

The stability diagram for a given tune distribution is obtained by solving the corresponding dispersion relation. An approximate dispersion relation for betatron detuning with longitudinal amplitude has been derived by J. Scott Berg and F. Ruggiero [9]

$$
\begin{aligned}
\Delta Q_{\mathrm{coh}}^{-1} & =\frac{1}{\mathcal{N}} \iiint_{0}^{\infty} \frac{J_{z}^{|m|} \Psi_{0}(\mathbf{J})}{Q-Q_{u}\left(J_{z}\right)-m Q_{s}} d^{3} \mathbf{J}, \quad u \in\{x, y\}, \\
\mathcal{N} & =\iiint_{0}^{\infty} J_{z}^{|m|} \Psi_{0}(\mathbf{J}) d^{3} \mathbf{J} .
\end{aligned}
$$

$\mathbf{J}=\left(J_{x}, J_{y}, J_{z}\right)$ is the vector of particle actions in all three dimensions, $Q_{u}\left(J_{z}\right)=Q_{0, u}+\Delta Q_{u}\left(J_{z}\right)$ describes the tune
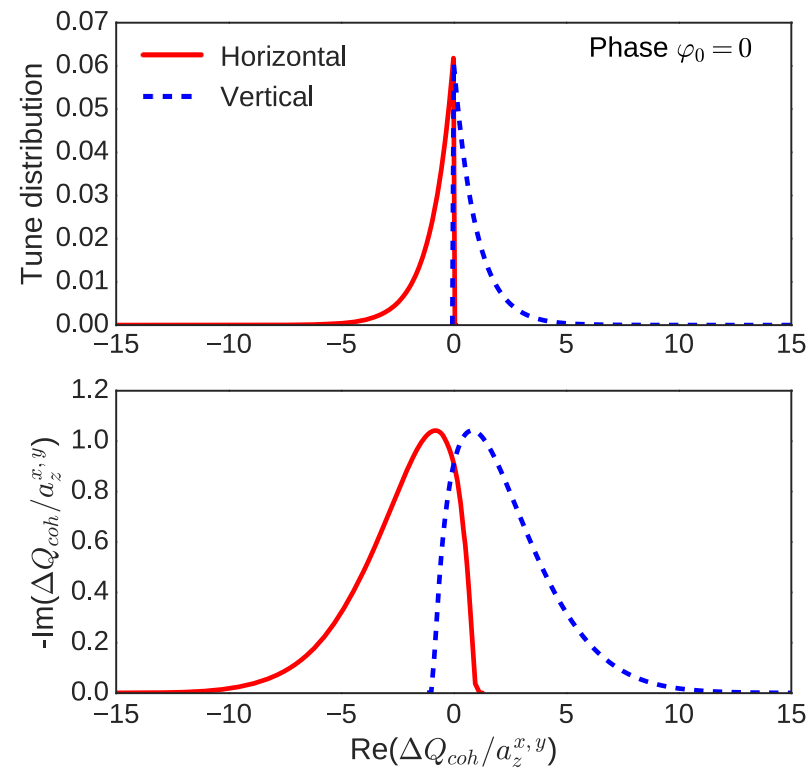

FIG. 6. Top: Incoherent tune distributions obtained analytically for a Gaussian beam with a single rf quadrupole with a phase of $\varphi_{0}=0$ assuming $\beta_{x}=\beta_{y}$. Bottom: Corresponding stability diagrams determined by solving Eq. (9) numerically for an azimuthal mode $m=0$ head-tail instability. 
dependence on $J_{z}, m$ is the azimuthal mode number of the instability under consideration, and $\Psi_{0}$ represents the unperturbed particle distribution. The stability diagram is obtained by numerically computing the tune shifts $\Delta Q_{\text {coh }}$ for different values of $Q$. According to the Landau bypass rule, a small complex part $i \epsilon$ is added to the denominator of Eq. (9) to perform the integration [3]. An example of stability diagrams in the two transverse planes is shown in the bottom plot of Fig. 6 for the corresponding tune distributions displayed in the top plot. An azimuthal mode $m=0$ head-tail instability is assumed here. The way to read the plots is that all the coherent instabilities with an unperturbed $\Delta Q_{\text {coh }}$ (i.e., in the absence of any tune spread) situated below the line traced out by the stability diagram will be Landau damped. In absence of Landau damping only the modes with $-\operatorname{Im}\left(\Delta Q_{\text {coh }}\right) \leq 0$ would be stable, i.e. the stability diagram in that case would be represented by a constant function with $-\operatorname{Im}\left(\Delta Q_{\text {coh }}\right)=0$. It is clear that the asymmetry of the tune spreads is reflected in the shape of the stability diagrams. This has important consequences for the stabilizing efficiency of the rf quadrupole in the two transverse planes as further discussed in Sec. V.

The dispersion relation in Eq. (9) is based on two main assumptions and does hence not describe all the beam dynamics aspects in presence of an rf quadrupole. First, in the general case it would depend on the specific impedance of the machine under consideration. In the derivation made by Scott Berg and Ruggiero, this dependency has been neglected under the assumption that the frequency range of the impedance is small compared to the frequency spectrum of the beam [9]. This condition holds for instance for a strongly peaked impedance, i.e., a narrow-band resonator with a very high quality factor. However, for the impedance model and the beam parameters of a hadron collider like the LHC for example, it is no longer valid. One reason is that several machine components have an impedance that extends over a large range of frequencies [18]. Second, an rf quadrupole acts on the betatron tunes as a function of the longitudinal coordinate and thus introduces a correlation between the betatron and the synchrotron motion. By consequence, it directly affects the interaction between the beam and the impedance with the result that the effective impedance changes [4]. This effect is similar to a chromaticity which can be accounted for by the head-tail phase parameter describing the frequency shift of the beam spectrum with respect to the impedance. A change of the effective impedance leads to a modification of the complex coherent tune shift of the instability under consideration. This manifests itself for example as a change of the instability rise time which can become faster or slower depending on the impedance of the machine and the respective unstable head-tail mode. At large enough rf quadrupole strengths it is even possible that a mode is excited on a different synchrotron side band (change of the azimuthal mode number) [20,21].

\section{PYHEADTAIL}

\section{A. Basic concepts}

PyHEADTAIL is a 6D macroparticle tracking code under development at CERN [10]. It is the successor of the well-established HEADTAIL code [22]. The purpose of the software is to accurately and effectively model the formation of collective instabilities in circular particle accelerators to make the study of the involved mechanisms possible, and to develop and evaluate appropriate mitigation techniques.

The basic PyHEADTAIL model represents a particle bunch as a collection of macroparticles, each of which is described by a mass, an electric charge, and its generalised coordinates and canonically conjugate momenta with two longitudinal and four transverse dimensions. The particles can be initialised in various distributions, amongst others Gaussian, waterbag, or airbag, also including the possibility for longitudinal matching to a multiharmonic rf bucket.

The accelerator ring is divided into a number of segments. At the beginning and at the end of every segment there is an interaction point where the macroparticles experience collective effects (wake fields/beam coupling impedance, electron-cloud, space-charge, etc.) or kicks from a specific accelerator component, such as a transverse feedback or an rf quadrupole. The betatron motion between two consecutive interaction points is modeled with linear maps which take into account the Twiss parameters and the dispersion at the start and at the end of the connecting segment. Non-linear tracking features such as chromaticity, i.e. the variation of the betatron tune with the relative momentum deviation, are modeled as a change in the phase advance of each individual macroparticle in the beam. The betatron detuning with transverse amplitude introduced by magnetic octupoles for example is parametrized by the effective machine anharmonicities and is implemented in the same manner. The synchrotron motion is either linear or nonlinear, potentially including particle acceleration and the effects of multiharmonic rf systems.

\section{B. Rf quadrupole models and benchmarks}

The rf quadrupole is implemented in PyHEADTAIL with two different models: (i) as a detuning element, and (ii) as localized kicks. The first model directly applies Eq. (4) and is implemented equivalently to other effective nonlinear tracking features, such as chromaticity and magnetic octupoles. Every macroparticle experiences a change in its phase advance depending on its longitudinal position in the bunch. In the second, more accurate localized kick model, every macroparticle receives kicks to its transverse and longitudinal momenta computed according to the thin-lens approximation defined by Eqs. (1) and (2). Betatron detuning then follows as a result after transverse tracking. The use of the thin-lens approximation is justified as the focal length of an $\mathrm{rf}$ quadrupole is much larger than the 
length of the device itself [23]. For example, the strength $b^{(2)}$ required to stabilize the most unstable head-tail mode in the HL-LHC at $7 \mathrm{TeV}$ is of the order of $0.3 \mathrm{Tm} / \mathrm{m}$ (see Sec. V C). This translates into a focal length $f>7.7 \times 10^{4} \mathrm{~m}$ which is orders of magnitude larger than the length of the device.

Both the detuning and the localized kick model of the rf quadrupole account for the constant phase offset $\varphi_{0}$ introduced in Sec. II. That way, different operational modes of the device can be studied. In terms of collective effects and Landau damping in particular, the two models are expected to produce identical results. The main difference is that the detuning model does not apply kicks to the particle momenta, meaning that the transverse and longitudinal action of each individual macroparticle remain unchanged. By consequence, resonances potentially introduced by an rf quadrupole cannot be excited and must instead be analyzed with the localized kick model. The two models are hence particularly useful when coherent and incoherent effects from an rf quadrupole are to be studied separately.

The results of a benchmark comparing the two rf quadrupole models in PyHEADTAIL are displayed in Fig. 7. The rf quadrupole phase is set to $\varphi_{0}=0$. The two histograms show the incoherent vertical tune distributions established after tracking a 6D Gaussian bunch with $50^{\prime} 000$ macroparticles over five synchrotron periods assuming linear synchrotron motion. The frequency analysis is performed by means of the sussix code [24]. The two models yield consistent results within expectations given the limited number of macroparticles. The bin colored in green represents particles at $1 \sigma$, i.e. with a longitudinal action of $J_{z}=\sigma_{z}^{2} / 2 \beta_{z}$. The blue dashed line marks the corresponding detuning expected from analytical

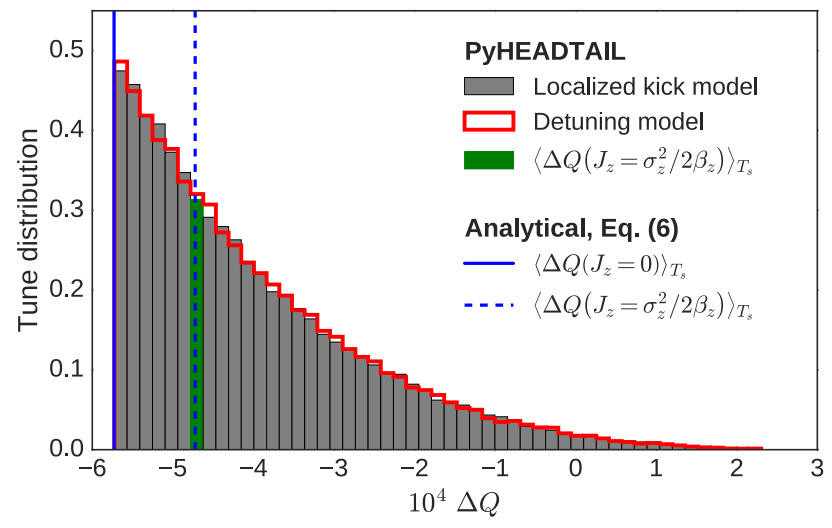

FIG. 7. Comparison of the incoherent betatron tune distributions introduced by an $\mathrm{rf}$ quadrupole for the detuning (red) and the localized kick (grey) model implemented in PyHEADTAIL. Both models give consistent results. The detuning obtained from PyHEADTAIL for particles with respectively $J_{z}=\sigma_{z}^{2} / 2 \beta_{z}$ (green) and $J_{z}=0$ is in good agreement with analytical calculations (blue). calculations [Eq. (6)] and shows an excellent agreement with both tracking models. The approximation of Eq. (6) holds as the beam and rf quadrupole parameters are chosen such that $\omega \sigma_{z} / \beta c \ll 1$. The blue solid line marks the average betatron detuning determined analytically for particles at $J_{z}=0$, i.e., particles residing in the center of the longitudinal phase space. As expected, it coincides with the lower bound of the distribution. Since for these particles $z$ remains zero over time, the (average) detuning is given simply by the constant quadrupolar term $-\beta_{y} b^{(2)} / 4 \pi B_{0} \rho$. This result is exact independent of whether or not the condition $\omega \sigma_{z} / \beta c \ll 1$ holds.

\section{NUMERICAL PROOF OF CONCEPT}

The numerical validation of the stabilizing effect of an rf quadrupole is based on a horizontal single-bunch instability originally observed experimentally in the LHC at $3.5 \mathrm{TeV}$ during machine commissioning in 2010 [11]. Dedicated studies in the LHC demonstrated that this particular instability is characterized as a slow head-tail mode with an azimuthal mode number $m=-1$. Furthermore, it was clearly shown experimentally that it can be suppressed by means of the Landau damping mechanism introduced by powering the Landau octupoles installed in the machine. The focusing and defocusing octupole families are powered with the same current, but opposite signs, i.e., $I_{\mathrm{oct}, f}=-I_{\mathrm{oct}, d}$, if not stated otherwise. The threshold current in the Landau octupoles required to Landau damp the head-tail mode was determined to be $I_{\text {oct }, f}^{\text {Exp }}=-15 \pm 5 \mathrm{~A}$. The corresponding instability rise time was measured to be $\tau \approx 9.8 \mathrm{~s}$ at $I_{\text {oct }, f}=-10 \mathrm{~A}$. The corresponding rise time at $I_{\mathrm{oct}, f}=0 \mathrm{~A}$ is expected to be significantly faster, but is not accessible from experimental measurements.

To confirm the validity of the numerical modeling, the observations made in the LHC are reproduced using two different accelerator physics models. First, the 6D macroparticle tracking code PyHEADTAIL, and second, a circulant matrix model (CMM) [25] implemented in the BimBim code [26]. The main input for both codes is an accurate impedance model of the LHC as well as the beam and machine parameters at the time of the measurement. They are summarized in Table I.

TABLE I. Main parameters used in PyHEADTAIL and BimBim to reproduce the LHC experimental machine setup at 3.5 TeV [11].

\begin{tabular}{lcc}
\hline \hline Parameter & Symbol & Value \\
\hline Bunch intensity & $\mathrm{N}_{b}$ & $1.0 \times 10^{11} \mathrm{p}^{+} / \mathrm{b}$ \\
Beam energy & $E$ & $3.5 \mathrm{TeV}$ \\
Chromaticity & $Q_{x, y}^{\prime}$ & 6 \\
Transverse normalized emittance & $\epsilon_{x, y}$ & $3.75 \mu \mathrm{m} \mathrm{rad}$ \\
Bunch length & $\sigma_{z}$ & $0.06 \mathrm{~m}$ \\
\hline \hline
\end{tabular}



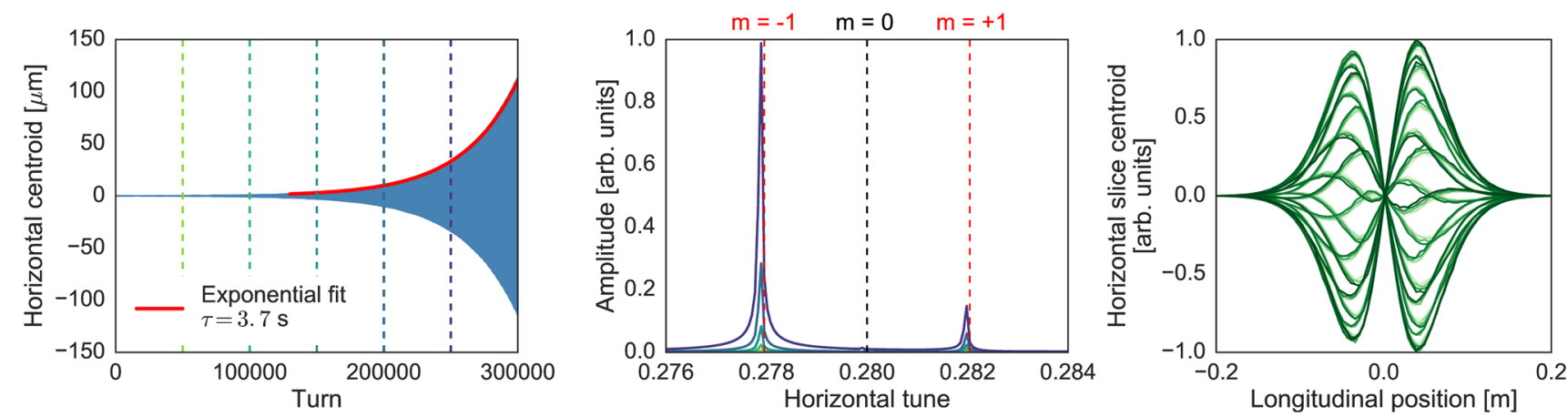

FIG. 8. Left: Horizontal bunch centroid evolution over time with an exponential fit of the rise time (red). Middle: Frequency spectra of the bunch centroid at different times during the simulation (see dashed lines in the first plot). The most unstable mode is an azimuthal mode $m=-1$ in agreement with the experiments. Right: Horizontal slice centroid vs. longitudinal position for 80 consecutive turns. The head-tail motion is characterized by one node in the center of the bunch.

\section{A. Stabilization from Landau octupoles}

PyHEADTAIL simulations make a full characterization of the observed unstable mode possible. The main results are summarized in Fig. 8 where $10^{6}$ macroparticles have been tracked over $3 \times 10^{5}$ turns. The first plot (left) shows the time evolution of the horizontal bunch centroid in absence of incoherent tune spread. The amplitude growth is fitted with an exponential function $f(t)=\exp (t / \tau)$ to obtain the rise time $\tau=3.7 \mathrm{~s}$ of the unstable mode. For comparison with the above-mentioned experimental measurements, the rise times from simulations are also determined for two different nonzero Landau octupole currents. At $I_{\text {oct }, f}=$ $-10 \mathrm{~A}$ the rise time is $\tau_{10}=4.5 \mathrm{~s}$ and at $I_{\mathrm{oct}, f}=-15 \mathrm{~A}$ it is $\tau_{15}=12.8 \mathrm{~s}$. The values are consistent with the experiments. A spectral analysis of the bunch centroid oscillation at different times during the evolution of the instability is shown in the middle plot. The spectral curves correspond to the vertical dashed lines in the first plot and are obtained from a fast-Fourier-transform with a window size of $16^{\prime} 384$ turns. The spectra clearly show that the azimuthal mode $m=-1$ is the fastest growing mode, which is again consistent with experimental observations. The corresponding head-tail mode pattern is a standing wave with a single node in the bunch center (right plot) indicating a radial mode number 1 . The characterization of the head-tail instability at hand is completed by the complex coherent tune shift $\Delta Q_{\text {coh }}$ of the unperturbed mode, i.e., in absence of Landau damping. The real part is obtained from spectral analysis (SUSSIX), and the imaginary component is calculated from the instability rise time fit. The results are

$$
\begin{aligned}
& \operatorname{Re}\left(\Delta Q_{\mathrm{coh}}^{\mathrm{PyHT}}\right)=-3.6 \times 10^{-6}, \\
& \operatorname{Im}\left(\Delta Q_{\mathrm{coh}}^{\mathrm{PyHT}}\right)=-9.2 \times 10^{-5} .
\end{aligned}
$$

To determine the amount of Landau damping required to suppress this particular mode, the LHC Landau octupoles are added to the PyHEADTAIL tracking model and a scan in $I_{\mathrm{oct}, f}$ is performed with a stepsize of $5 \mathrm{~A}$. A selection of the results is presented in Fig. 9. The instability is suppressed with a Landau octupole current of $I_{\mathrm{oct}, f}^{\mathrm{PyHT}}=-17.5 \pm 2.5 \mathrm{~A}$ which is in excellent agreement with experimental measurements.

Complementary to the studies carried out with PyHEADTAIL, the second accelerator physics model used here is the CMM implemented in the BimBim code. It includes amongst others the effects of impedance and chromaticity, but neglects for instance multiturn wake fields and Landau damping from magnetic octupoles [27]. The former is a valid approximation for the wake fields present in the LHC as they decay on a time scale faster than the revolution period [18]. The absence of Landau damping in the CMM is a consequence of the linearization of the system in the transverse planes. This approximation excludes the possibility to account for the nonlinear effects required to model detuning with transverse amplitude. BimBim computes directly the unperturbed $\Delta Q_{\text {coh }}$ by numerically solving an eigenvalue equation. For the LHC instability, the most unstable mode in BimBim is again the azimuthal mode $m=-1$ with a coherent tune shift of

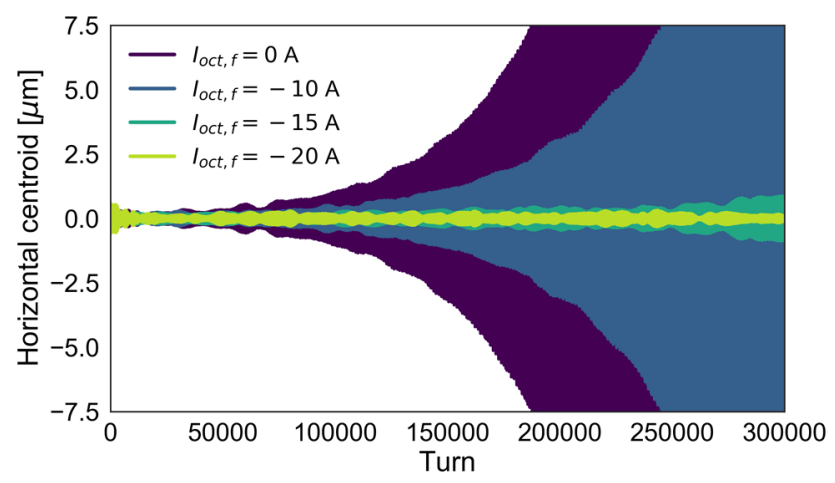

FIG. 9. PyHEADTAIL results of the bunch centroid motion over $3 \times 10^{5}$ turns in the LHC for different values of the currents in the Landau octupole magnets. 


$$
\begin{aligned}
& \operatorname{Re}\left(\Delta Q_{\mathrm{coh}}^{B B}\right)=-3.9 \times 10^{-6}, \\
& \operatorname{Im}\left(\Delta Q_{\mathrm{coh}}^{B B}\right)=-9.6 \times 10^{-5} .
\end{aligned}
$$

The values are in good agreement with PyHEADTAIL tracking simulations.

As the CMM does not model Landau damping from magnetic octupoles, a different method must be chosen instead to determine the current $I_{\mathrm{oct}, f}$ required for beam stability. A popular one is to use stability diagram theory for Landau octupoles [9]. The Python solver for stability diagrams (PySSD) is used here for that purpose [26,28]. The stability diagrams from LHC Landau octupoles are shown in Fig. 10 in the complex tune space $\operatorname{Re}\left(\Delta Q_{\text {coh }}\right)$ vs. $-\operatorname{Im}\left(\Delta Q_{\text {coh }}\right)$ for two different Landau octupole currents, $I_{\mathrm{oct}, f}=-15 \pm 5 \mathrm{~A} \quad$ (green), and $I_{\mathrm{oct}, f}=-17.5 \pm 1 \mathrm{~A}$ (orange). The colored areas are respectively the corresponding uncertainties or the resolution of the scan in Landau octupole current. The former corresponds to the experimental measurements, while the latter is determined from the unperturbed complex coherent tune shifts (BimBim and PyHEADTAIL). The values of $\Delta Q_{\text {coh }}$ of the unperturbed mode as obtained from BimBim and PyHEADTAIL are given by the red and blue markers respectively. For this particular case, the theory predicts stability from the Landau octupoles at $I_{\mathrm{oct}, f}^{\mathrm{SDT}}=-17.5 \pm 1 \mathrm{~A}$ as illustrated in Fig. 10 by the orange dashed line and area. This is again fully consistent with tracking simulations and experiments (green line and area).

The studies presented above clearly show that the impedance model of the LHC used for numerical simulations with PyHEADTAIL and BimBim is reliable. Both accelerator physics models produce the same outcome and manage to reproduce the observations made in the machine to a high degree of accuracy. The observed

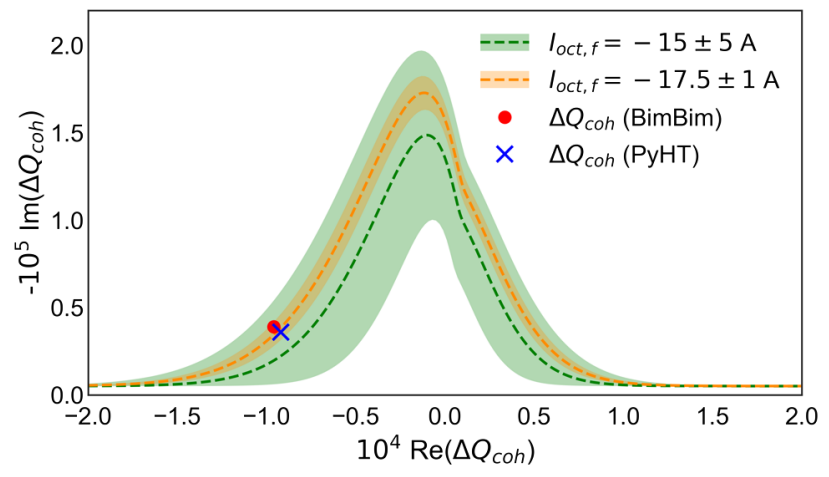

FIG. 10. The dashed lines are stability diagrams obtained with the PySSD code for two different Landau octupole currents $I_{\mathrm{oct}, f}=-15 \pm 5 \mathrm{~A}$ (green) and $I_{\mathrm{oct}, f}=-17.5 \pm 1 \mathrm{~A}$ (orange). The colored areas are the corresponding uncertainties. The former corresponds to the values obtained experimentally, while the latter is determined from the unperturbed complex coherent tune shifts (red circle: BimBim, blue cross: PyHEADTAIL). head-tail mode is Landau damped in experiments, tracking simulations, and in theory with fully consistent results on the required Landau octupole current. The fact that stability diagram theory predicts the same Landau octupole current as obtained from experiments and tracking underlines that the stabilization of this mode is indeed a result of Landau damping. Furthermore, the Landau damping mechanism is accurately modeled in PyHEADTAIL and this forms the basis for the numerical proof of concept of the stabilizing effect from an rf quadrupole discussed in the following.

\section{B. Stabilization from an rf quadrupole}

The PyHEADTAIL study presented above is repeated with now an rf quadrupole (localized kick model) instead of the LHC Landau octupoles. A scan is performed in the integrated quadrupolar field strength $b^{(2)}$ with the Landau octupoles switched off. The results of the scan are summarized in Fig. 11. They show a picture that is very similar to Fig. 9. With increasing quadrupolar strength, and hence increasing tune spread, the growth rate of the unstable mode is reduced and eventually suppressed entirely. The stability threshold is $b^{(2)}=0.105 \pm 0.005 \mathrm{Tm} / \mathrm{m}$. Although not displayed here, an identical result is obtained with the detuning model of the rf quadrupole in PyHEADTAIL. The studies confirm the effect expected from the theory discussed in Sec. II D, and demonstrate that an rf quadrupole for beam stabilization can work conceptually. According to preliminary cavity design studies, the stabilizing quadrupolar strength required here could be achieved with a single cavity with an equivalent active length of about $0.1 \mathrm{~m}$ [12]. For comparison, to stabilize the same mode with the LHC Landau octupoles an equivalent active length of about $1.5 \mathrm{~m}$ is required. This is assuming that they are powered at their maximum current of $550 \mathrm{~A}$.

In analogy to the analysis performed above for the Landau octupoles, the stabilizing strength of an rf quadrupole is analyzed also by means of stability diagram theory. For that purpose, the approximate dispersion relation in

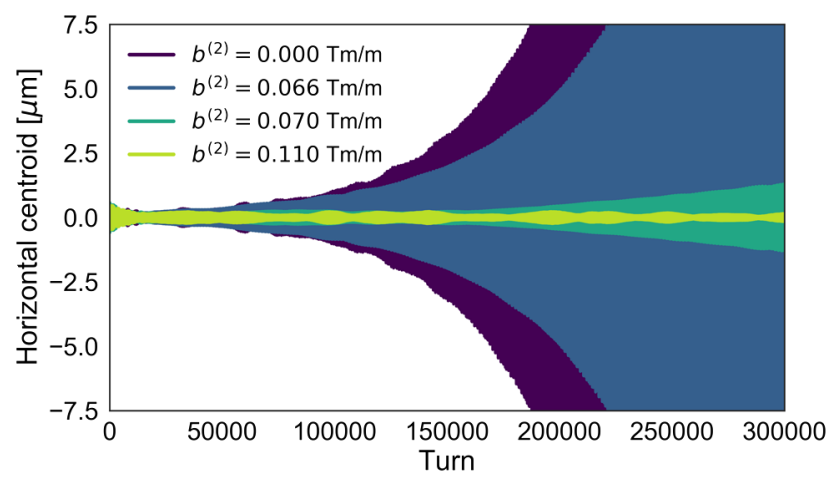

FIG. 11. PyHEADTAIL results of the bunch centroid motion over $3 \times 10^{5}$ turns in the LHC for different values of the rf quadrupole strength $b^{(2)}$. 


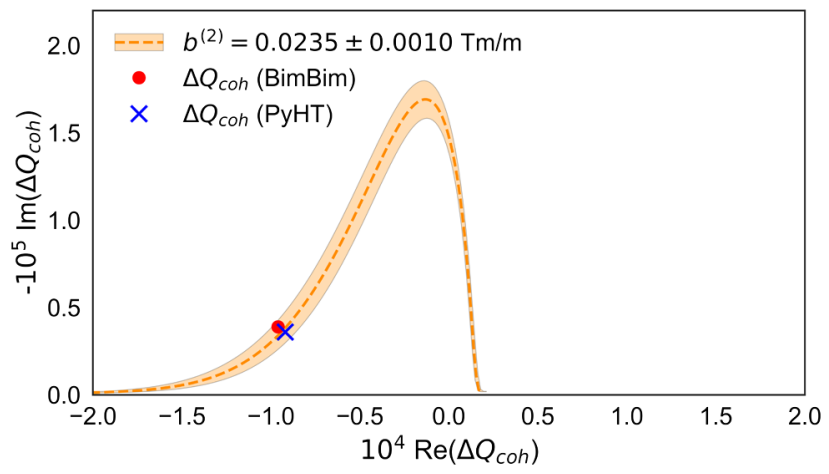

FIG. 12. The dashed line is the stability diagram obtained by numerically solving the approximate dispersion relation in Eq. (9) for an rf quadrupole with $b^{(2)}=0.0235 \pm 0.0010 \mathrm{Tm} / \mathrm{m}$. The colored area is the corresponding resolution of the scan in $b^{(2)}$. The unperturbed complex coherent tune shifts from BimBim (red circle) and from PyHEADTAIL (blue cross) are shown as well.

Eq. (9) is solved numerically for the parameters of the PyHEADTAIL simulation. The outcome is presented in Fig. 12. The red and blue markers are again the complex coherent tune shifts obtained with the BimBim code and PyHEADTAIL respectively. The dashed line marks the stability diagram for an $\mathrm{rf}$ quadrupole strength of $b^{(2)}=0.0235 \pm 0.0010 \mathrm{Tm} / \mathrm{m}$. The colored area corresponds to the resolution of the scan in $b^{(2)}$. There is a clear discrepancy of a factor four in the required stabilizing strength between theory and PyHEADTAIL tracking simulations. The main reason for this difference is that the dispersion relation does not take into account the impedance model of the LHC, as explained in Sec. II D. It assumes instead that there is only a single peak in the impedance spectrum that is responsible for driving the given instability [9]. Clearly, this is not the case here as (i) the LHC impedance has several broad-band components, and (ii) the overlap between the bunch spectrum and the impedance determines the coherent tune shift (and growth rate) of the instability $[4,18]$. Furthermore, the tracking simulation takes into account the full cosine dependence of the detuning from the rf quadrupole, while the analytical formula only considers the first order term in $J_{z}$ [see Eq. (7)]. For the given rf quadrupole frequency and bunch length, the detuning of the particles at large amplitudes $J_{z}$, and thus the overall tune spread and the Landau damping, are overestimated compared to the tracking simulations.

Comparing the amounts of RMS tune spread required from magnetic octupoles and an $\mathrm{rf}$ quadrupole helps to estimate the Landau damping efficiency of the two mitigation techniques. The RMS tune spreads required to suppress slow head-tail instabilities are typically much smaller than the synchrotron tune $Q_{s}$ since $\operatorname{Re}\left(\Delta Q_{\text {coh }}\right) \ll Q_{s}$. For the unstable mode studied here, they are (from PyHEADTAIL)

$$
\begin{aligned}
& \Delta Q_{\mathrm{rms}}^{\mathrm{oct}}=(2.4 \pm 0.3) \times 10^{-5} \approx 0.012 Q_{s}, \\
& \Delta Q_{\mathrm{rms}}^{\mathrm{rfq}}=(3.4 \pm 0.5) \times 10^{-5} \approx 0.017 Q_{s},
\end{aligned}
$$

with $Q_{s} \approx 2 \times 10^{-3}$. Although the two values are of a similar order of magnitude, the discrepancy is significant and emphasizes that the amount of incoherent tune spread alone does not give the entire picture about whether an unstable mode is Landau damped or not. The imaginary part of $\Delta Q_{\text {coh }}$ as well as the shape of the stability diagram are both essential ingredients to address this question. This becomes clear by comparing Figs. 10 and 12. The reason for the difference of the required tune spreads is a consequence mainly of the way the Landau damping mechanism works for detuning with transverse and longitudinal amplitude respectively. The dispersion integral equations for the two approaches differ significantly from each other [9]. As a result, the corresponding stability diagrams can have a different shape and the tune spreads required to suppress a particular instability will in general be different as well.

\section{ALTERNATIVE SCHEMES}

Stability diagram theory for detuning with longitudinal amplitude predicts amongst others a strong asymmetry in the stabilizing efficiencies between the two transverse planes. This is studied in the following by qualitatively comparing theory against tracking simulations. To overcome the asymmetry, a two-family scheme for rf quadrupoles is proposed and the performance is validated with tracking simulations. The idea is motivated by the twofamily scheme employed for beam stabilization with magnetic octupoles [17]. Finally, the synergy between rf quadrupoles and magnetic octupoles is studied in terms of stabilizing efficiency for an HL-LHC machine setup.

\section{A. Qualitative comparison with PyHEADTAIL simulations}

The comparisons between stability diagram theory and tracking simulations shown here are only of qualitative nature due to the simplifications made in the derivation of the dispersion relation (see Sec. II D). Nevertheless, they give insight into the mechanisms and are used to motivate a more advanced scheme of $\mathrm{rf}$ quadrupoles to improve the performance presented in Sec. V B.

The horizontal and the vertical complex coherent tune shifts of the unstable head-tail modes are typically very similar for a hadron collider like the LHC, i.e., $\Delta Q_{\text {coh }}^{x} \approx \Delta Q_{\text {coh }}^{y}$. For LHC operational beam and machine parameters for instance the most unstable impedance-driven head-tail modes are characterized by $\operatorname{Re}\left(\Delta Q_{\text {coh }}^{x, y}\right)<0$. For a single rf quadrupole, the stability diagrams in Fig. 6 illustrate that the stable regions in the two transverse planes have different shapes. Given that $\operatorname{Re}\left(\Delta Q_{\text {coh }}^{x, y}\right)<0$, one plane (here: horizontal) is expected to exhibit a much better stabilizing 
efficiency than the other one for an rf quadrupole with a phase of $\varphi_{0}=0$. The situation is inverse for $\varphi_{0}=\pi$. This asymmetry has first been observed in PyHEADTAIL simulations for the LHC instability at an energy of $3.5 \mathrm{TeV}$, introduced in Sec. IV. For this particular case, by changing the phase $\varphi_{0}$ from 0 to $\pi$, the stabilizing quadrupolar strength increases roughly by a factor three from $b_{0}^{(2)}=0.105 \pm 0.005 \mathrm{Tm} / \mathrm{m}$ to $b_{\pi}^{(2)}=0.35 \pm 0.02 \mathrm{Tm} / \mathrm{m}$.

The asymmetric nature of the rf quadrupole kicks is impractical, and a systematic study is made with PyHEADTAIL to further examine this particular observation and to find possible solutions to circumvent it. To simplify the simulation set-up, a pure dipolar analytical broad-band resonator impedance (quality factor of $Q=1$ ) is used with identical components in the horizontal and the vertical planes. For further simplification of the setup, the chromaticity is set to $Q^{\prime}<0$ to induce a weak azimuthal mode $m=0$ head-tail instability (note that the study is done above transition energy) [4]. This has no impact on the underlying stabilizing mechanism described by stability diagram theory. Both the real and the imaginary components of the coherent tune shift $\Delta Q_{\text {coh }}^{x, y}$ are controlled by varying the beam intensity. With increasing intensity, the coherent tune shift becomes larger due to the stronger interaction of the beam with the impedance. It is hence expected from theory that more rf quadrupole strength will be required for stabilization. The two transverse planes are affected equivalently by the intensity increase. However, stability diagram theory predicts that the stabilizing rf quadrupole strength must be different in the two planes due to the strong asymmetry of the stability diagrams (see Fig. 6, bottom).

The results of the simulation studies are summarized in Fig. 13. $80^{\prime} 000$ macroparticles have been tracked over $10^{5}$ turns and a scan in $b^{(2)}$ has been performed for each beam intensity setting. The top plot corresponds to an $\mathrm{rf}$ quadrupole with $\varphi_{0}=0$ and clearly represents the asymmetry expected from the theory. The horizontal plane becomes stable already at much lower quadrupolar strength than the vertical one. By changing $\varphi_{0}$ from 0 to $\pi$, the behaviour in the two planes is swapped (bottom plot), again in accordance with expectations given the quadrupolar nature of the device. At the maximum beam intensity, the difference between the threshold quadrupolar strengths in the two planes reaches almost a factor five for this particular case.

Figure 14 underlines the qualitative agreement between tracking simulations and theory and is an explanation for the observations made. The plot shows the complex coherent tune space with the dependence of the unperturbed $\Delta Q_{\text {coh }}^{x, y}$ on the beam intensity, represented by the colored markers. They are normalized to the absolute value of the maximum real coherent tune shift measured at the highest intensity of the scan. The real and the imaginary parts

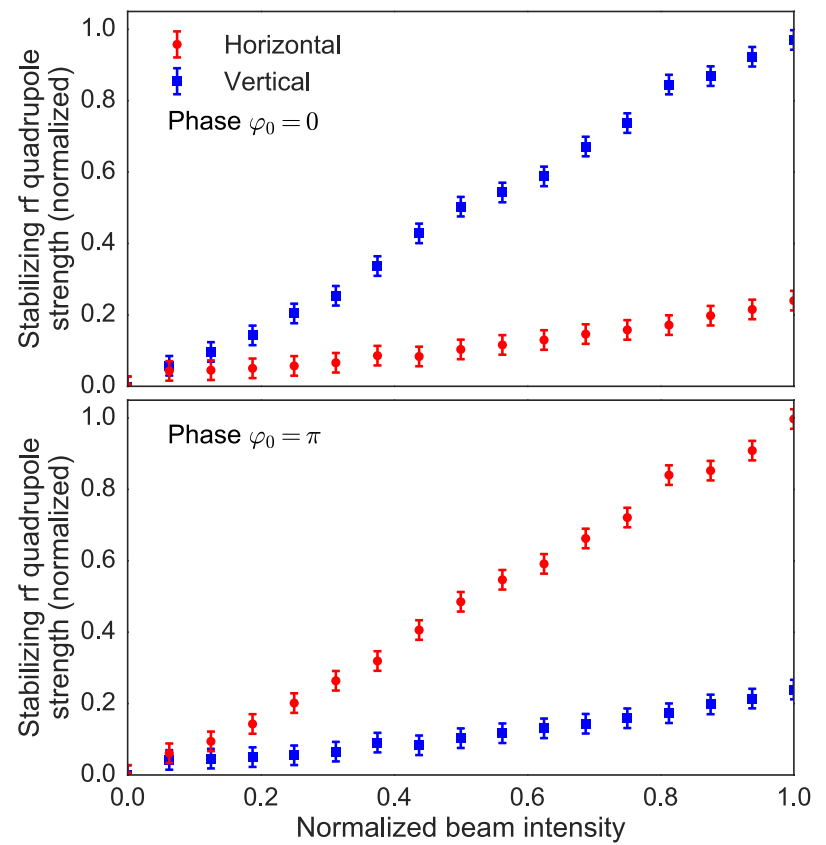

FIG. 13. PyHEADTAIL tracking simulations illustrating the stabilizing efficiency in the horizontal (red) and the vertical (blue) planes with an rf quadrupole with $\varphi_{0}=0$ (top) and $\varphi_{0}=\pi$ (bottom) respectively.

depend approximately linearly on the beam intensity. Furthermore, $\operatorname{Re}\left(\Delta Q_{\text {coh }}^{x, y}\right) \leq 0$. Stability diagrams for two different quadrupolar strengths of an rf quadrupole operating with $\varphi_{0}=0$ are overlaid for the horizontal (solid lines) and the vertical plane (dashed lines). It is obvious that the stabilizing behavior in the horizontal plane is expected to be significantly better than in the vertical one. Additionally, comparing the stability diagrams for the two quadrupolar strengths, the stable area for the horizontal plane grows

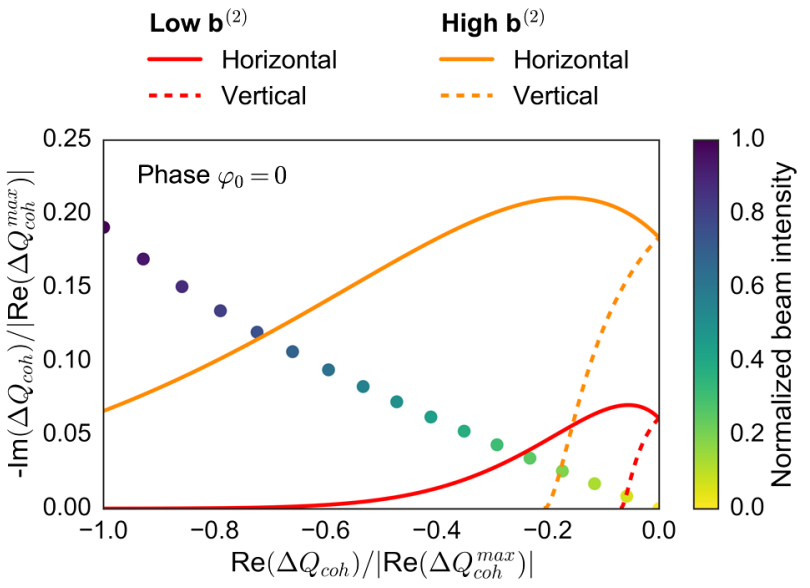

FIG. 14. Evolution of the unperturbed coherent tune shift in the complex tune space as a function of intensity. Stability diagrams for the horizontal (solid lines) and the vertical (dashed lines) planes are overlaid for two different strengths for an $\mathrm{rf}$ quadrupole operating with $\varphi_{0}=0$. 
much faster than that in the vertical plane. This explains the increase in the difference between the stability thresholds as a function of beam intensity shown in Fig. 13.

\section{B. Two-family scheme}

The asymmetric stabilizing behavior in the two transverse planes can be overcome by installing two independent rf quadrupole families that operate with opposite phases and are placed at two different locations in the machine lattice. One of them is placed at high $\beta_{x}$, low $\beta_{y}$ to improve beam stability mainly in the horizontal plane, while the other one is installed at a location with low $\beta_{x}$ and high $\beta_{y}$ for stability mainly in the vertical plane. Naturally, the difference between the local beta functions must be as large as possible to avoid (significant) compensation of the detuning effect from the other if quadrupole family.

Figure 15 illustrates the stabilizing efficiency in the two transverse planes for the two-family rf quadrupole scheme for the broad-band resonator driven instability introduced in Sec. VA. For illustrative purposes, values of ten have been assumed for respectively the ratio $\beta_{x} / \beta_{y}$ and $\beta_{y} / \beta_{x}$ at the two rf quadrupole locations. The vertical axis represents the total quadrupolar strength, i.e., the sum of the strengths of the two families, to allow a better comparison with the one-family schemes depicted in Fig. 13. The two families are powered with the same strengths. There are two main obvious advantages. First, the strong asymmetry between the two planes is removed and the required stabilizing strength is now equal for both planes. Second, the total quadrupolar strength to achieve stability in both planes is significantly lower overall. However, since the two families counteract each other to a small amount, the stabilizing strength in the two-family scheme cannot reach the values of the respective favored plane in the one-family scheme.

\section{Synergy with magnetic octupoles}

To understand the practicality and usefulness of an $\mathrm{rf}$ quadrupole in a future hadron collider, it is important to assess its interplay with magnetic octupoles. The study

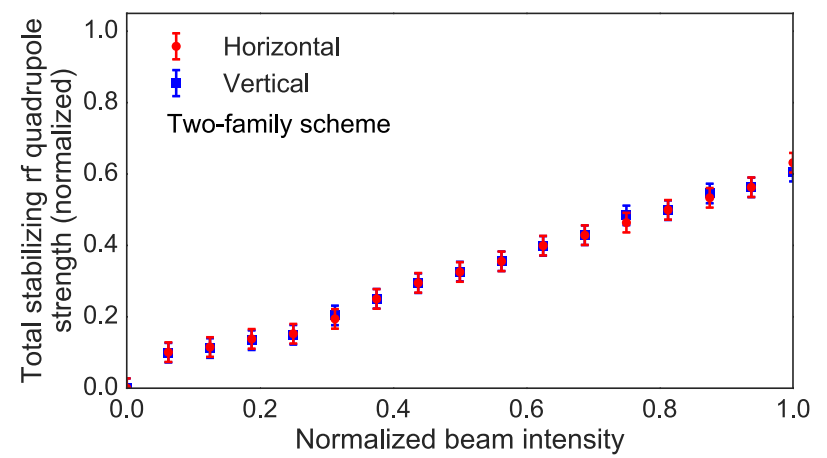

FIG. 15. PyHEADTAIL tracking simulations illustrating the stabilizing efficiency in the horizontal (red) and the vertical (blue) planes with the two-family rf quadrupole scheme.
TABLE II. Main machine and simulation parameters used for the HL-LHC studies with PyHEADTAIL.

\begin{tabular}{lcc}
\hline \hline Parameter & Symbol & Value \\
\hline Bunch intensity & $\mathrm{N}_{b}$ & $2.2 \times 10^{11} \mathrm{p}^{+} / \mathrm{b}$ \\
Beam energy & $E$ & $7 \mathrm{TeV}$ \\
Chromaticity & $Q_{x, y}^{\prime}$ & 10 \\
Transverse normalized emittance & $\epsilon_{x, y}$ & $2.5 \mu \mathrm{m} \mathrm{rad}$ \\
Bunch length & $\sigma_{z}$ & $0.082 \mathrm{~m}$ \\
\hline \hline
\end{tabular}

presented here is based on the HL-LHC. The stabilizing performance of an ensemble of $800 \mathrm{MHz}$ superconducting rf quadrupole cavities is evaluated in presence of the LHC Landau octupoles. The latter will remain an important part of the instability mitigation toolset also for the HL-LHC. A single-bunch instability driven by the dipolar accelerator impedance for foreseen operational flat-top beam and machine parameters serves as a study case. The main values of the machine set-up are summarized in Table II. An idealised bunch-by-bunch transverse feedback system with a damping time of 50 turns is included in the simulation model. At a chromaticity of $Q_{x, y}^{\prime}=10$ the most unstable mode is a slow head-tail instability with azimuthal and radial mode numbers of 0 and 2 respectively [29]. This is consistent with experimental observations made in the LHC at $6.5 \mathrm{TeV}[6]$.

PyHEADTAIL simulations with $8 \times 10^{5}$ macroparticles tracked over $6 \times 10^{5}$ turns predict that beam stability from LHC Landau octupoles alone is achieved for $I_{\text {oct }, f}=170 \pm 10$ A. This is about one third of their maximum current. By adding an rf quadrupole to the simulation model, the stabilizing current in the Landau octupoles can be significantly lowered as demonstrated in Fig. 16. The beta functions at the potential location of the rf quadrupole in the HL-LHC are set to $\beta_{x, y}=200 \mathrm{~m}$ (conservative). Although there is a minor effect on the threshold current $I_{\text {oct }, f}$ up to a cavity strength of about $b^{(2)}=0.1 \mathrm{Tm} / \mathrm{m}$, the simulations predict that already for $b^{(2)} \geq 0.27 \mathrm{Tm} / \mathrm{m}$ the Landau

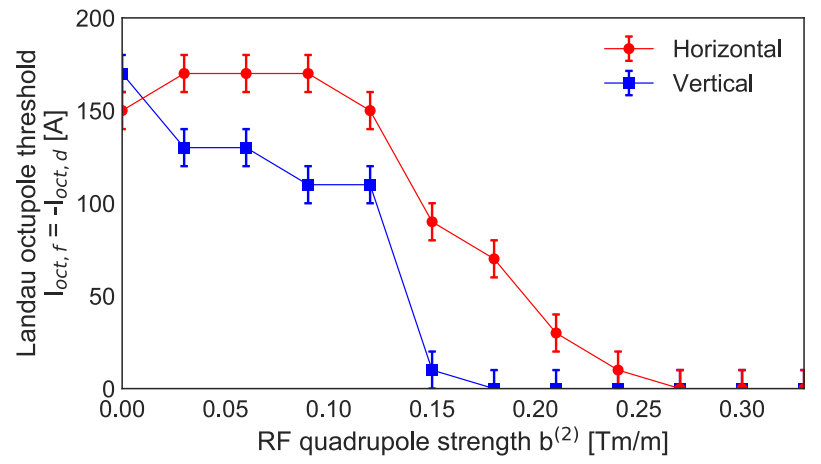

FIG. 16. PyHEADTAIL tracking simulations showing the combined stabilizing effect from LHC Landau octupoles and an rf quadrupole in the HL-LHC. 
octupoles are no longer required to mitigate the instability. Given the preliminary cavity design, the latter strength can be provided with about one to two rf cavities [12].

\section{SUMMARY}

The working principle of an rf quadrupole for Landau damping has been recapped and potential advantages over conventional stabilizing methods, in particular magnetic octupoles, have been pointed out. The major one is that the incoherent betatron tune spread is dependent on the longitudinal action spread which is several orders of magnitude larger than the transverse ones for the beams of LHC, HL-LHC, and FCC-hh. This method provides hence a much larger handle for producing the incoherent tune spread needed for Landau damping. The required active length of rf quadrupole compared to that of magnetic octupoles is greatly reduced, even if the field strengths in an rf quadrupole are much lower than those in magnetic octupoles. The advantage is particularly pronounced at higher beam energies as the RMS tune spread from an $\mathrm{rf}$ quadrupole decays only with $1 / \gamma$ compared to $1 / \gamma^{2}$ for magnetic octupoles.

Two models of an rf quadrupole have been implemented in the PyHEADTAIL 6D macroparticle tracking code, and were successfully benchmarked against each other and against analytical calculations. The validation of the PyHEADTAIL model, i.e., the formation of impedance-driven instabilities as well as the Landau damping mechanism, was based on a slow head-tail mode studied experimentally at $3.5 \mathrm{TeV}$ in the LHC. The comparison of the LHC Landau octupole current required for beam stability revealed an excellent agreement between experiment, numerical simulations from particle tracking and the circulant matrix model, and stability diagram theory. The Landau octupoles were replaced with an rf quadrupole in a second step to show that such a device can provide stability in a similar manner. The comparison with stability diagram theory for rf quadrupoles shows, however, that more work is necessary to improve the theoretical predictions.

Due to the quadrupolar nature of the device, the stabilizing efficiency in the two transverse planes is strongly asymmetric. This is predicted by stability diagram theory and is confirmed by tracking simulations. Based on these results, a two-family scheme for rf quadrupoles was proposed and tested numerically. Not only could the asymmetry be cleared away, but also the overall quadrupolar strength required for beam stability was reduced. Finally, based on an HL-LHC operational scenario, the combined stabilizing effect from magnetic octupoles and an rf quadrupole was studied and shown to be successful.

\section{OUTLOOK AND CLOSING REMARKS}

While the results are promising in terms of the mitigation of impedance-driven collective instabilities, further studies are required to assess the usefulness and practicality of an $\mathrm{rf}$ quadrupole. Among them are (i) incoherent effects including resonances and dynamic aperture, (ii) the efficiency of the device against other types of collective instabilities, e.g., induced by the presence of an electron-cloud, and (iii) the cavity alignment tolerances and the effects of a beam entering the cavity with a transverse offset. In parallel, methods are under study for an experimental validation of the collective effects simulations presented here. Although the construction and test of a prototype $\mathrm{rf}$ quadrupole cavity in an existing accelerator would be the most direct way to achieve this goal, it is associated with large costs and a time-scale of the order of years. As an alternative, stabilization from second order chromaticity $Q^{\prime \prime}$ is under study. $Q^{\prime \prime}$ provides betatron detuning with longitudinal amplitude, equivalently to an rf quadrupole in a first order approximation [21]. The main advantage of $Q^{\prime \prime}$ over the rf quadrupole is that it can be introduced in an existing machine like the LHC for instance by powering the installed main sextupole magnets in a specific scheme. Measurements have already been performed in the LHC with single nominal bunches at an energy of $6.5 \mathrm{TeV}$ [20]. The preliminary analysis of the experiments shows that (i) $Q^{\prime \prime}$ indeed contributes to beam stability and (ii) PyHEADTAIL accurately models the involved mechanisms. A more detailed analysis will be reported at a later date.

\section{ACKNOWLEDGMENTS}

The authors would like to acknowledge Gianluigi Arduini, Xavier Buffat, Lee Carver, Antoine Maillard, Elias Métral, and Giovanni Rumolo for important contributions to these studies.

[1] G. Apollinari, I. Béjar Alonso, O. Brüning, M. Lamont, and L. Rossi, CERN Yellow Report No. CERN-2015-005, 2015.

[2] M. Benedikt and F. Zimmermann, CERN Report No. CERN-ACC-2015-0164, 2015.

[3] L. Landau, On the vibrations of the electronic plasma, $\mathrm{Zh}$. Eksp. Teor. Fiz. 16, 574 (1946) [J. Phys. USSR 10, 25 (1946)].

[4] A. Chao, Physics of Collective Beam Instabilities in High Energy Accelerators, Wiley Series in Beam Physics and Accelerator Technology (Wiley, New York, 1993).

[5] O. S. Brüning, P. Collier, P. Lebrun, S. Myers, R. Ostojic, J. Poole, and P. Proudlock, CERN Yellow Report No. CERN2004-003-V-1, 2004.

[6] L. Carver et al., Current Status of Instability Threshold Measurements in the LHC at $6.5 \mathrm{TeV}$, in Proc. of International Particle Accelerator Conference (IPAC'16), Busan, Korea, 2016, International Particle Accelerator Conference No. 7 (JACoW, Geneva, Switzerland, 2016), p. 1434, TUPMW011.

[7] E. Métral et al., Measurement and Interpretation of Transverse Beam Instabilities in the CERN Large Hadron 
Collider (LHC) and Extrapolations to HL-LHC, in Proc. of ICFA Advanced Beam Dynamics Workshop on HighIntensity and High-Brightness Hadron Beams (HB'16), Malmö, Sweden, 2016, ICFA Advanced Beam Dynamics Workshop on High-Intensity and High-Brightness Hadron Beams No. 57 (JACoW, Geneva, Switzerland, 2016), p. 254, TUAM2X01.

[8] A. Grudiev, Radio frequency quadrupole for Landau damping in accelerators, Phys. Rev. ST Accel. Beams 17, 011001 (2014).

[9] J. S. Berg and F. Ruggiero, Stability diagrams for Landau damping, in Proceedings of the Particle Accelerator Conference, Vancouver, BC, Canada, 1997 (IEEE, New York, 1997), Vol. 2, p. 1712.

[10] E. Métral et al., Beam Instabilities in Hadron Synchrotrons, IEEE Trans. Nucl. Sci. 63, 1001 (2016).

[11] E. Métral, B. Salvant, and N. Mounet, CERN Report No. CERN-ATS-2011-102, 2011.

[12] K. Papke and A. Grudiev, Design of an rf quadrupole for Landau damping, Phys. Rev. Accel. Beams 20, 082001 (2017).

[13] M. Kapchinski and V. A. Tepliakov, Linear Ion Accelerator with Spatially Homogeneous Strong Focusing, Prib. Tekh. Eksp. 19 (1970).

[14] S. Peggs and T. Satogata, Introduction to Accelerator Dynamics (Cambridge University Press, Cambridge, England, 2017).

[15] V. V. Danilov, Increasing the transverse mode coupling instability threshold by RF quadrupole, Phys. Rev. ST Accel. Beams 1, 041301 (1998).

[16] E. A. Perevedentsev and A. A. Valishev, Synchrobetatron dynamics with a radio-frequency quadrupole, in Proceedings of the 8th European Particle Accelerator Conference, Paris, 2002 (EPS-IGA and CERN, Geneva, 2002), p. 1574.

[17] J. Gareyte, J.-P. Koutchouk, and F. Ruggiero, CERN Report No. LHC-Project-Report-91, 1997.

[18] N. Mounet, Ph.D. thesis, SB, Lausanne, 2012.

[19] P. Baudrenghien and T. Mastoridis, Longitudinal emittance blowup in the large hadron collider, Nucl. Instrum. Methods Phys. Res., Sect. A 726, 181 (2013).
[20] M. Schenk, D. Amorim, N. Biancacci, X. Buffat, L. Carver, R. D. Maria, K. Li, E. Métral, and B. Salvant, Practical Stabilisation of Transverse Collective Instabilities with Second Order Chromaticity in the LHC, in Proc. of International Particle Accelerator Conference (IPAC'17), Copenhagen, Denmark, 2017, International Particle Accelerator Conference No. 8 (JACoW, Geneva, Switzerland, 2017), p. 4477, THPVA026.

[21] M. Schenk, X. Buffat, L. Carver, A. Grudiev, K. Li, A. Maillard, E. Métral, and K. Papke, RF Quadrupole Structures for Transverse Landau Damping in Circular Accelerators, in Proc. of International Particle Accelerator Conference (IPAC'17), Copenhagen, Denmark, 1419 May, 2017, International Particle Accelerator Conference No. 8 (JACoW, Geneva, Switzerland, 2017), p. 2516, WEOAB3.

[22] G. Rumolo and F. Zimmermann, Electron cloud simulations: beam instabilities and wakefields, Phys. Rev. ST Accel. Beams 5, 121002 (2002).

[23] H. Wiedemann, Particle Accelerator Physics, 4th ed. (Springer, Berlin, 2015).

[24] R. Bartolini and F. Schmidt, CERN Report No. SL-Note98-017-AP, 1998.

[25] V. V. Danilov and E. A. Perevedentsev, Feedback system for elimination of the transverse mode coupling instability, Nucl. Instrum. Methods Phys. Res., Sect. A 391, 77 (1997).

[26] X. Buffat, Ph.D. thesis, SB, Lausanne, 2015.

[27] S. White, X. Buffat, N. Mounet, and T. Pieloni, Transverse mode coupling instability of colliding beams, Phys. Rev. ST Accel. Beams 17, 041002 (2014).

[28] X. Buffat, W. Herr, N. Mounet, T. Pieloni, and S. White, Stability diagrams of colliding beams in the Large Hadron Collider, Phys. Rev. ST Accel. Beams 17, 111002 (2014).

[29] M. Schenk, A. Grudiev, K. Li, and K. Papke, Use of RF Quadrupole Structures to Enhance Stability in Accelerator Rings, in Proc. of ICFA Advanced Beam Dynamics Workshop on High-Intensity and High-Brightness Hadron Beams (HB'16), Malmö, Sweden, 2016, THPM7X01; ICFA Advanced Beam Dynamics Workshop on HighIntensity and High-Brightness Hadron Beams No. 57 (JACoW, Geneva, Switzerland, 2016), p. 505. 